\title{
Sciences infirmières et douleur : des soins, des savoirs, de la recherche et de l'enseignement
}

\author{
C. Berlemont \\ (C) Lavoisier SAS 2022
}

Depuis toujours, la profession infirmière évolue avec la société, avec les avancées de la médecine, des autres sciences comme les sciences humaines et bien sûr au rythme des apports de la recherche en santé.

En France, l'avancement du statut d'infirmière prend un virage à la fin du XIX ${ }^{\mathrm{e}}$ siècle en se structurant comme profession grâce à une formation scientifique et à la création du diplôme d'État infirmier. D'activités en soins « charitables », de soins en « secondant assidûment et docilement le médecin », elle devient une profession qui va s'autonomiser au fil des années. Ce métier en pleine évolution va développer ses compétences, s'adapter et répondre aux exigences, aux besoins de santé de la population tout en cherchant une reconnaissance sociale.

Cette profession est « jeune ». Rappelons-nous : d'une nouvelle définition de l'infirmier(ière) en 1970 vers la reconnaissance d'un rôle propre en 1978 et le début de la recherche clinique en soins infirmiers en 1980. D'ailleurs, depuis ces années jusqu'en 2019, la profession poursuit sa quête d'une reconnaissance sociale (avec le développement de son autonomie, l'apparition des règles professionnelles, le diagnostic infirmier, les décrets d'actes et enfin la prescription infirmière). Elle s'ouvre aux techniques, s'émancipe de la profession médicale, crée ses propres structures et s'intègre maintenant au monde universitaire.

Que d'avancées avec la reconnaissance des infirmier(ière)s de pratiques avancées (IPA) et la création de la section « sciences infirmières » au Conseil national des universités.

Les infirmier(ière)s qui exercent spécifiquement en structure douleur et/ou en mission transversale ont bien entendu suivi aussi l'évolution de la profession et de la discipline infirmière. Si leur rôle au début se limitait à la réalisation de soins sur prescription médicale, très rapidement ils/elles vont développer leur expertise clinique et structurer leurs savoirs et activités faisant émerger l'identité de l'infirmier(ière) ressource douleur (IRD). L'organisation et la composition des structures douleur (SDC) a permis aux IRD de se construire avec le

C. Berlemont $(\bowtie)$

Infirmière ressource douleur et formatrice

e-mail : cae.berlemont@gmail.com soutien des médecins algologues et des psychologues exerçant dans ces structures. Il est possible de suivre cette évolution grâce aux référentiels réalisés par les IRDS : un référentiel d'activités (2007), puis un référentiel d'activités de consultation clinique infirmière (2016), suivi du référentiel d'activités et de compétences (2020) et enfin un argumentaire pour la reconnaissance d'une pratique avancée à l'exercice de l'IRD au sein des SDC (2021) que je vous invite vivement à lire (téléchargeable sur le site de la SFETD).

Dans cet argumentaire, est proposée une comparaison entre la fonction IRD et celle des IPA. La conclusion est sans appel : les IRD ont développé des compétences et des activités de haut niveau.

Poursuivre, encourager le développement de la recherche infirmière en France reste encore un pas à faire. Il manque des filières universitaires, des laboratoires de recherche et de la restructuration (création de postes infirmiers pour la recherche par exemple).

Ce dossier vous permet d'avoir un aperçu de cette évolution et de la contribution qu'apportent les IRD à la population et à la communauté de la santé.

Trois articles « de terrain » très différents, mais où il sera question de relation et de communication. Le témoignage d'un aidant naturel accompagnant son proche âgé, co-écrit par Brigitte Hérisson et moi-même, où vous pourrez vous interroger sur l'importance les liens, de la communication et du travail d'équipe, puis deux articles complémentaires de Karine Constans et Dominique Gillet autour du parcours de soin du patient douloureux chronique, qui mettent en lumière l'importance des liens entre les acteurs de soin pour accompagner ce patient.

Ensuite, un article autour de l'exercice infirmier et de la place de la formation de Pascale Thibault-Wanquet permet de mieux cerner les enjeux de l'enseignement autour de la thématique douleur et fait écho à l'article témoignage.

Puis nous entrerons plus spécifiquement dans le champ des IRD avec un article de nos collègues belges, Nadine Chard'homme et Valérie Marteau, sur la classification des interventions en soins infirmiers. Un travail qui permet d'apprécier l'ampleur des interventions des IRD (ou infirmiers algologues) dans leur pratique quotidienne et qui se met en 
parallèle avec les référentiels de la commission professionnelle infirmière de la SFETD.

Et parce que 2018-2019 est marquée par l'arrivée des IPA, deux articles complémentaires de Sophie Vanderheyden et Amélie Segers pour la Belgique, et Esther MolinaBeltran et Isabelle Da Costa pour la France.

Pour terminer, un article de Christophe Debout sur la contribution infirmière à l'évaluation et au traitement du patient douloureux et l'apport des sciences infirmières qui permet d'ouvrir les perspectives d'avenir des sciences infirmières en douleur.

Que ces lectures puissent vous apporter et vous montrer l'importance de la discipline infirmière appliquée au domaine de la douleur. Et que, dans quelques années, nous puissions entendre nos premiers docteurs en sciences infirmières et douleur. 